\title{
Sub-nanometer precision modification of the optical properties of three-dimensional polymer-based photonic crystals
}

\author{
Georg von Freymann ${ }^{\mathrm{a}, \mathrm{b}, *}$, Timothy Y.M. Chan ${ }^{\mathrm{a}}$, Sajeev John ${ }^{\mathrm{a}}$, \\ Vladimir Kitaev ${ }^{\mathrm{b}}$, Geoffrey A. Ozin ${ }^{\mathrm{b}}$, Markus Deubel ${ }^{\mathrm{c}}$, Martin Wegener ${ }^{\mathrm{d}}$ \\ ${ }^{\mathrm{a}}$ Department of Physics, University of Toronto, 60 St. George Street, \\ Toronto, ON, Canada M5S 1 A7 \\ ${ }^{\mathrm{b}}$ Materials Chemistry Research Group, Department of Chemistry, University of Toronto, \\ 80 St. George Street, Toronto, ON, Canada M5S 3 H6 \\ ${ }^{\mathrm{c}}$ Institut für Nanotechnologie, Forschungszentrum Karlsruhe in der Helmholtzgemeinschaft, 76021 Karlsruhe, Germany \\ ${ }^{\mathrm{d}}$ Institut für Angewandte Physik, Universität Karlsruhe (TH), 76128 Karlsruhe, Germany \\ Received 12 August 2004; received in revised form 28 September 2004; accepted 19 October 2004 \\ Available online 11 November 2004
}

\begin{abstract}
We present a convenient post-fabrication technique to precisely tune the optical properties of polymer-based threedimensional photonic crystals with sub-nanometer precision. Conventional air-plasma etching is utilized to modify the filling fraction of direct laser written polymer photonic crystals beyond the wavelength-imposed limits. Tuning of the optical properties is monitored in transmission and reflection spectroscopy. A simple model for the etching process is proposed and found to be in good agreement with the experimental observations.
\end{abstract}

(C) 2004 Elsevier B.V. All rights reserved.

PACS: 42.70.Qs; 42.25.Dd; 42.25.Fx; 81.05.Zx

Keywords: 3D photonic crystals; Tuning of optical properties; Plasma etching

\section{Introduction}

Scientists, involved in the quest of making largescale three-dimensional (3D) photonic crystals [1,2],

\footnotetext{
* Corresponding author. Tel.: +1 4169784233 ; fax: +1 4169712011 .

E-mail address: freymann@physics.utoronto.ca (G. von Freymann).
}

have recently turned their attention to templating 3D photonic crystals using polymer materials. Lithographic techniques like laser holography $[3,4]$ and direct laser writing [5,6] allow for the fabrication of 3D photonic crystals up to square millimeter size without incorporating intrinsic defects, commonly found when using colloidal templating techniques (see for a discussion of different intrinsic defect types [7]). These optical lithography methods, when used in

1569-4410/\$ - see front matter (C) 2004 Elsevier B.V. All rights reserved.

doi:10.1016/j.photonics.2004.10.001 
combination, allow for the incorporation of waveguides and resonators and therefore enable direct templating of functional photonic devices. They have only one drawback: the minimal size of structural features is connected to the wavelength of the illuminating laser and/or limited by the resolution achievable with the polymer materials. This limitation might hinder the direct use of these templates for the fabrication of 3D photonic crystals at telecommunication wavelengths via infiltration of high-index of refraction materials like silicon [6]. Therefore, a technique to overcome this limitation is required but should not only yield the desired result but equally be suited for massproduction.

Plasma etching is a universal technological tool for surface patterning and modification. The underlying operation principle is quite simple: radio-frequency is utilized to excite gaseous argon, nitrogen, oxygen or air, which creates a plasma. This plasma is a partially ionized complex gas composed of electrons, ions, atoms, free radicals and photons of various energies. The plasma produces an energized gas flow that modifies the physicochemical characteristics of solid surfaces without affecting the underlying bulk properties of the material. In particular, residues of organic materials are removed within seconds from the surface. But what will happen, if the underlying bulk material is a complex 3D photonic crystal made of an organic polymer, where the plasma ignites in the air voids but cannot create strong gas flows due to the complexity of the structure?

In this article, we utilize air-plasma etching to tune the optical properties of 3D polymer-based photonic crystals by changing the filling fraction of the polymer material. We show that feature sizes, well below the wavelength-imposed writing limitations, can be reached and that this plasma tuning allows, in principle, for sub-nanometer precision. The article is organized as follows: in Section 2, we describe the sample preparation and the applied conditions for the plasma etching. In Section 3, the change of the optical properties under plasma etching is explained. A simple numerical model for the etching process is proposed in Section 4, which in combination with bandstructure calculations supports our experimental observations. In Section 5, certain control experiments are presented, before we conclude the article in Section 6.

\section{Sample preparation}

The samples under investigation are lithographically written in commercially available negative photo resist SU-8 by a two-photon absorption direct laser writing technique [6]. To write 3D photonic crystal structures into SU-8 films a regeneratively amplified Ti:sapphire laser system is used with a pulse duration of $120 \mathrm{fs}$. The repetition rate can be computer controlled from $1 \mathrm{kHz}$ to single shot mode. The wavelength is tuned to $800 \mathrm{~nm}$, where the one-photon absorption of the resist is negligible. The output beam is attenuated by a half-wave plate/polarizer combination, and after beam expansion, typically $50 \mathrm{~nJ}$ of single pulse energy are coupled into an inverted microscope. There, the femtosecond pulses are focused into the resist by a $100 \times$ oil immersion objective with high numerical aperture $(\mathrm{NA}=1.4)$. The sample is placed on a capacitively controlled three-axis piezo scanning stage, which is operated in closed loop and provides a resolution of better than $5 \mathrm{~nm}$ at a full scanning range of $200 \mu \mathrm{m} \times 200 \mu \mathrm{m} \times$ $20 \mu \mathrm{m}$. A computer controls the scanning operation of the piezo and synchronizes its movements with the output of the laser system. The high intensity in the focal point allows for the two-photon absorption process. The exposed volumes (voxels) are of ellipsoidal shape with a ratio between axial diameter/lateral diameter of 2.7. The extraordinarily high quality of $3 \mathrm{D}$ photonic crystals fabricated with this method is documented elsewhere [6,8]. Although the method proposed in this article is suitable for all topologies of polymer-based photonic crystals, we concentrate on the well-known layer-by-layer or woodpile structure $[9,10]$, as changes in dimensions like rod diameter are easily accessible via scanning electron microscopy (SEM) and intuitively understandable.

For the plasma etching, we use a Harrick PDC-3XG plasma cleaner/sterilizer. The power levels applied to the radio frequency coils in our experiments are approximately $5.4 \mathrm{~W}$ and $2.0 \mathrm{~W}$. The airflow is controlled by a needle valve (Whithey SS-22RS2, set to 1.05) and is kept constant throughout all the experiments. An oil-roughing pump generates vacuum inside the sample chamber. The samples are placed in the center of the chamber and exposure times are measured with a stopwatch after plasma ignition. 
Fig. 1(a) shows a woodpile fabricated by direct laser writing. The lateral dimensions of the photonic crystal are $100 \mu \mathrm{m} \times 100 \mu \mathrm{m}$ and the thickness of the structure is 6 unit cells ( 24 rod layers). The massive bars at the sides stabilize the structure to reduce stressinduced deformation due to shrinkage during development. As all woodpiles are sputtered with $\mathrm{Pt}$ or $\mathrm{Au}$ prior to taking the SEM images (Hitachi S5200), all images in Fig. 1 are obtained using different woodpiles fabricated with exactly the same parameters. Fig. 1(b) presents a close-up of the top of a woodpile structure after 2 min plasma exposure. The lateral diameter of the rods is reduced to $227 \mathrm{~nm}$ from the original $270 \mathrm{~nm}$, while the integrity of the whole structure is preserved. After 5 min plasma exposure, the diameter of the topmost rods is already reduced to $143 \mathrm{~nm}$ [see Fig. 1(c)], while layers further down in the structure seem to be less attacked. This apparent inhomogeneity in the etching speed is a direct consequence of the air-polymer interface: the air plasma easily reaches the surface and flow rates are higher than deep in the bulk of the photonic crystal.

This anisotropy is even more obvious in Fig. 1(d) where it can be seen that after 20 min of plasma exposure the topmost layers are almost completely removed and just some uniformly shaped lumps are left. Fortunately, this pronounced inhomogeneity occurs just for the topmost layers - as expected from airflow considerations - and can be directly observed in the cross-section shown in Fig. 1(e). Even after prolonged plasma exposure (22 $\mathrm{min})$, the interior of the structure is intact and the diameter of the rods is reduced homogeneously. The obviously broken rods are a direct consequence of the mechanical sectioning process. The heavily attacked top layers can be clearly seen on the top of the structure. A rigorous analysis of the rod diameters from bottom to top reveals an average rod diameter of $200 \pm 20 \mathrm{~nm}$ for the 10 bottom most layers and a slight gradient of rod diameters down to $162 \pm 20 \mathrm{~nm}$ for the remaining rods.

\section{Optical characterization}

To show that the modification of the bulk properties of the photonic crystal is indeed isotropic we evaluate reflectance and transmittance spectra. The spectra are measured in a Fourier Transform Infrared Spectrometer (Hartmann-Braun Bomen) combined with an infrared microscope (SpectraTech, 15× Cassegrain objectives, $\mathrm{NA}=0.564$, aperture size $50 \mu \mathrm{m} \times$ $50 \mu \mathrm{m})$. The samples are aligned with their surfaces perpendicular to the optical axis, which corresponds to the $\Gamma X\left[\begin{array}{lll}0 & 0 & 1\end{array}\right]$ direction for fcc symmetry. Spectra are taken on the original sample subjected to consecutive exposures to the plasma in 1-min increments. Fig. 2 shows the evolution of the reflectance spectra for incrementally prolonged plasma exposure. The spectra are normalized to their maximum and vertically displaced for clarity. Prior to exposure (the topmost spectrum) a clear and prominent peak at about $1.75 \mu \mathrm{m}$ is observed. The side lobes on the blue side of the peak are due to the large opening angle of the Cassegrain objectives used in the FTIR microscope [6]. With increasing plasma exposure time (exposure time is given on the right axis), the main peak blue shifts as expected for a reduction in the filling fraction [11]. The shape of the spectra remains remarkably stable, even as the topmost layer is gradually removed for exposure times greater than $10 \mathrm{~min}$. Slight changes in the contrast of the side lobes as well as a small (4\%) reduction in the peak height might be explained with the change in the filling fraction and the resulting reduction of material contrast. This holds also for the transmittance spectra (not shown), which are even more sensitive to the bulk than the reflectance spectra (reflected light only effectively samples the top 3 unit cells (12 layers) of the structure. For transmission data showing the bulk influence, see Section 5). After $11 \mathrm{~min}$ of plasma exposure, the position of the reflectance peak is shifted by $80 \mathrm{~nm}$ to the blue [see dotted lines in Fig. 2].

The position of the maximum versus exposure time is depicted in the top part of Fig. 3. The open circles denote the peak position and the up and down triangles indicate the full width at half maximum (FWHM). A linear fit to these data (solid line) confirms the linear behavior and reveals the tuning rate of $6.9 \mathrm{~nm} / \mathrm{min}$. The slight broadening of the FWHM (1.4 nm/min or a change from $10 \%$ to $11 \%$ of the midgap wavelength over the whole etching time) is likely due to the gradient of the etching rate into the depth of the structure over the first few layers [Fig. 1(e)]. This leads to a corresponding gradient with depth (reminiscent of a chirped Bragg grating) in the solid volume-filling 

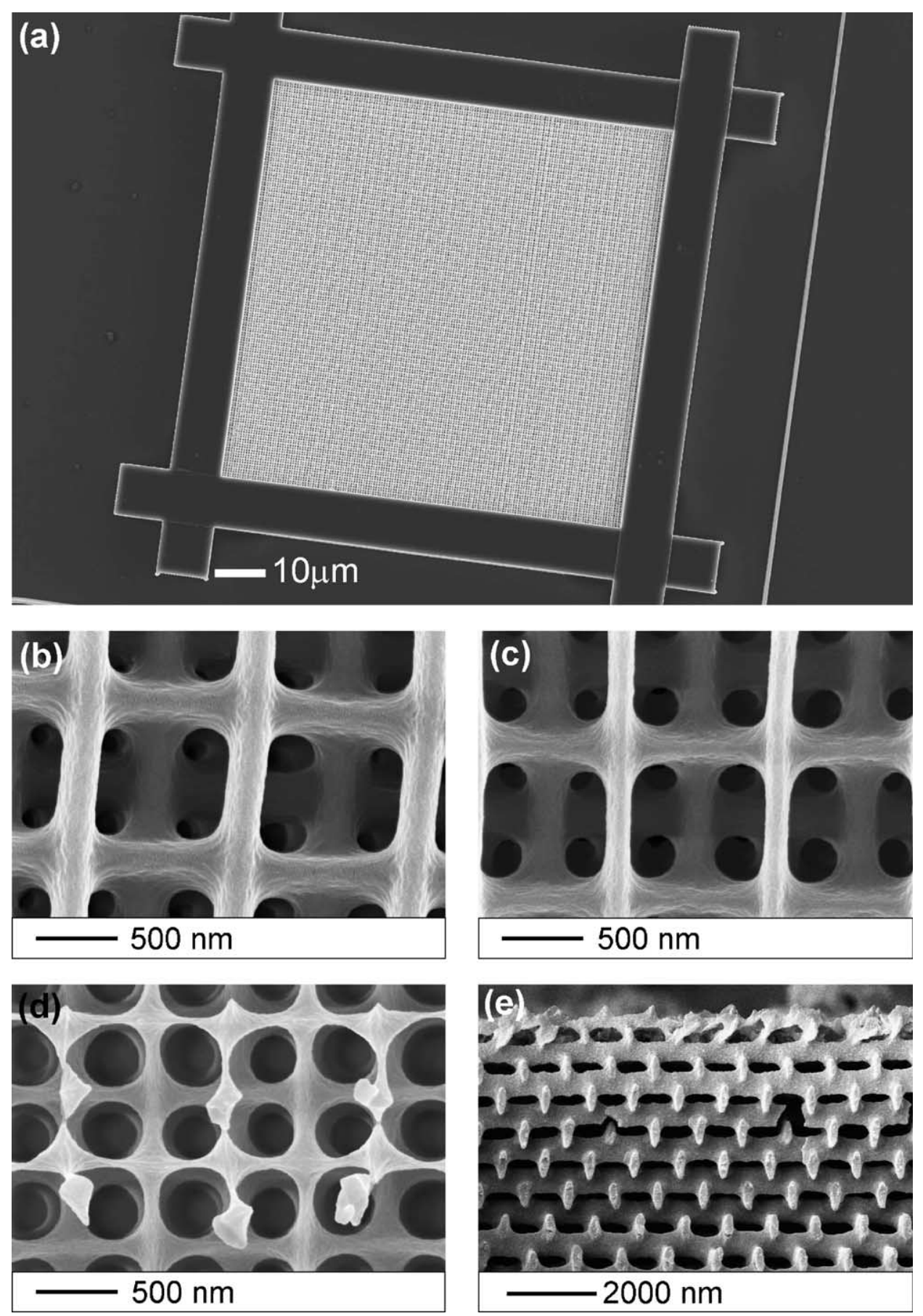

Fig. 1. Scanning electron micrographs of the woodpile structure under investigation: (a) top view of the whole structure. (b) Close up of the surface after 2 min plasma exposure. (c) Same as (b), but after 5 min exposure. The diameter of the rods is clearly reduced. (d) Same as (b) but after 10 min exposure. The topmost layer is strongly modified. (e) Cross-section of a woodpile taken after prolonged plasma exposure (22 min). The homogenous etching in the bulk can be clearly observed, while the topmost layers are heavily attacked. 


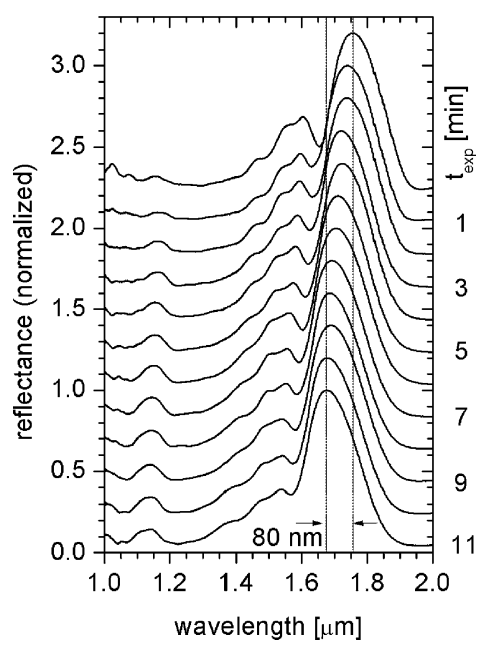

Fig. 2. Normalized reflectance spectra taken on the same woodpile structure subjected to gradually increasing exposure to oxygen plasma. The corresponding exposure times are given on the right hand side. With increased exposure time the position of the reflectance maximum blue shifts, while the overall shape of the spectrum stays almost the same. The spectra are normalized to the maximum and vertically displaced for better clarity.

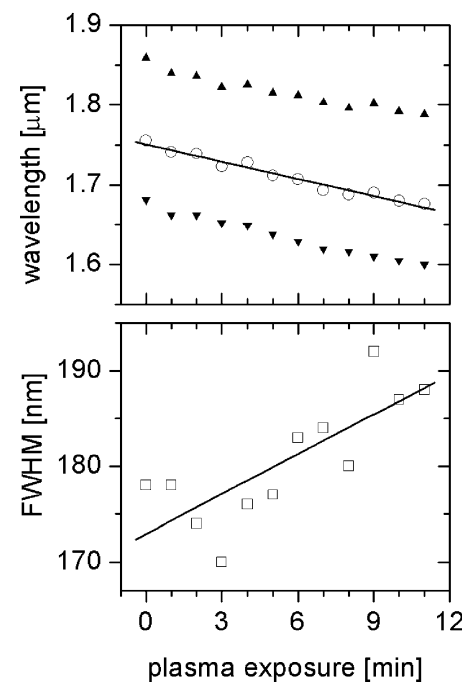

Fig. 3. Top: evolution of the spectral position of the reflectance maximum (open circles) under plasma exposure. The triangles represent the positions, where the peak height reaches $50 \%$. Bottom: evolution of the FWHM (open squares). The solid lines are linear fits to the data. fraction [see bottom part of Fig. 3]. In the presence of this gradient, the actual reflectivity FWHM is the union of FWHMs from different depths within the sample.

\section{Numerical simulations}

To support our experimental observations and to investigate in how far the structural changes during plasma exposure influence the actual bandstructure and therefore the optical properties of the sample, we implement a numerical model for the etching of the woodpiles. The model acts on one complete unit cell of the woodpile structure, taking into account the elliptical cross-section of the rods. To calculate the influence on the optical properties after a certain etching time, bandstructure calculations for photonic crystal built from the modified unit cell are performed. Assuming an isotropic etching process, the change of the volume $V$ filled with polymer with time can be described as:

$\frac{\delta V}{\delta t}=\int_{S} \gamma \mathrm{d} \vec{S}$

where $S$ is the surface of the structure exposed to the plasma and $\gamma$ is the etching rate. For simplicity, we assume that reactive species are supplied much faster than the etching process consumes them. For the simulations, the index of refraction of SU-8 after development $(n=1.6)$ was taken into account. The etching rate $\gamma=3.9 \mathrm{~nm} / \mathrm{min}$ gave the best fit to the experimental tuning curves. One unit cell of the structure was discretized with $128 \times 128 \times 181$ volume elements and structural changes were calculated in 6-s time intervals. Bandstructures for these unit cells were calculated using a plane wave expansion method.

Although it would be desirable to give a complete microscopic theory of the etching process for the whole structure, this is clearly beyond the scope of this article. Despite the inherent simplicity of our model, it perfectly describes all the experimental observations: Fig. 4(a) displays the change in volume filling fraction with exposure time (open circles). There are only minor deviations from a linear behavior (solid line). Fig. 4(b) shows bandstructure calculations along the 


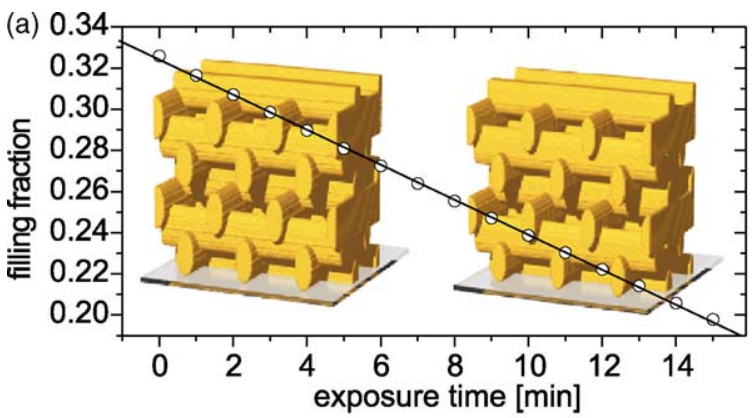

(b)

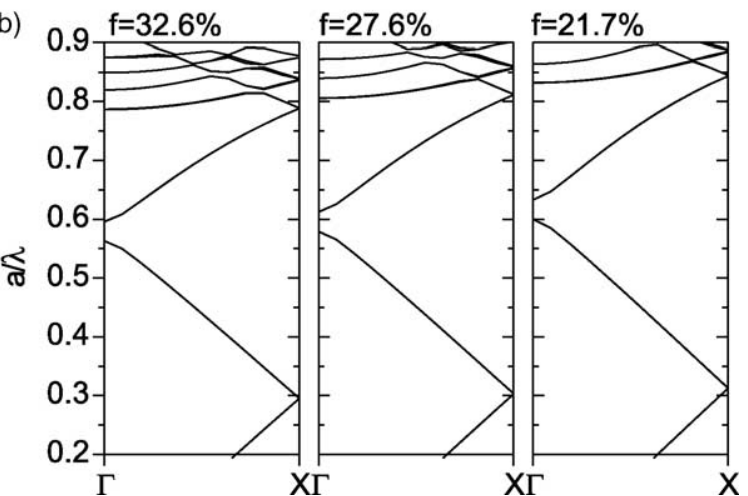

Fig. 4. Theory: (a) Reduction of the filling fraction with exposure time (open circles). A fitting reveals the almost linear behavior (solid line). The two images show the results of the theoretical modelling of the etching for the structure built of 12 unit cells each before etching (left) and after 13 min plasma exposure (right). (b) Bandstructure calculations for an infinite photonic crystal build from the unit cells modified by the etching simulation. The filling fractions correspond to no exposure (32.6\%), $6 \mathrm{~min}(27.6 \%)$, and $13 \mathrm{~min}$ (21.7\%) plasma exposure. A clear blue shift of the fundamental gap with almost no reduction in the width is observed. (For interpretation of the references to color in this figure legend, the reader is referred to the web version of this article.)

$\Gamma X$ (the $\left.\left[\begin{array}{lll}0 & 0 & 1\end{array}\right]\right)$ direction for three filling fractions. Again, the experimental observations are supported by theory: the midgap position shifts linearly with exposure time and the gap width stays almost constant. After tuning the midgap position nearly $100 \mathrm{~nm}$ to the blue, the gap width is only reduced by $0.2 \%$ as compared to the midgap wavelength. The influence of structural modifications on the optical properties seems to be negligible for the tuning range under consideration, as clearly visible changes in the unit cell show up for longer exposure times. Virtually unchanged bands at higher energies support this observation [see Fig. 4(b)].

\section{Control experiments}

To experimentally confirm the reproducibility of our observations, another woodpile photonic structure was fabricated and exposed to the plasma under exactly the same conditions. The resulting tuning rate of $6.5 \mathrm{~nm} / \mathrm{min}$ (not shown) confirms the high precision of this method. The small deviations from perfectly linear behavior can be attributed to slight inhomogeneities of plasma ignition in the plasma chamber and uncertainties of the exposure time and the airflow control.

For colloidal photonic crystals, it has been shown that the stop band position depends on the number of layers [12]. Taking into consideration the heavily attacked top layers, it is tempting to interpret the observed tuning phenomenon as a mere consequence of the removal of upper layers. To clarify this point, spectra of woodpiles fabricated with 22,21 , and 20 layers are shown from bottom to top in Fig. 5. The overall deviation in spectral position (marked with the dotted lines) is $24 \mathrm{~nm}$ and is due to the usual deviations during fabrication. This $24 \mathrm{~nm}$ is significantly smaller than the tuning depicted in Fig. 2, where we removed only one layer during plasma exposure.

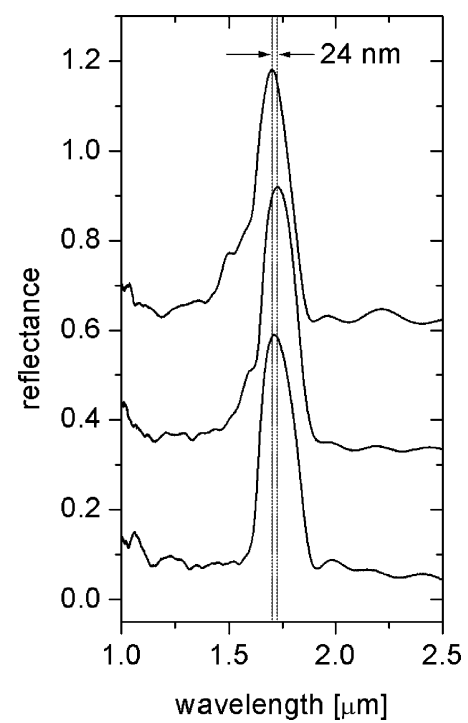

Fig. 5. Reflectance spectra of three different woodpiles, fabricated with the same parameters but different number of layers. From bottom to top: 22, 21, and 20 layers. The maximum difference in peak position is $24 \mathrm{~nm}$ and no blue shift with decreasing layer number is observed. Spectra are vertically displaced for clarity. 
Furthermore, there is no apparent blue shift with reduced number of layers, so that missing layers can be clearly excluded as the cause of the observed tuning.

To stress this point a little further, we repeated the tuning experiment with approximately half the radio frequency power. This slows down the tuning significantly. Reflectance and transmittance spectra were taken every $2 \mathrm{~min}$. The overall shift of the reflectance peak position is depicted in the top part of Fig. 6. Again, the tuning shows linear behavior (solid line), but - as expected - with almost half the tuning speed $(3.4 \mathrm{~nm} / \mathrm{min})$. By utilizing a computer-controlled plasma etching experiment, exposure times well below $1 \mathrm{~min}$ could be reached. This suggests the possibility of sub-nanometer precision tuning of the optical properties of polymer-based photonic crystals.

Although the tuning of the optical properties via the filling fraction is well understood, the removal speed for the topmost layers leaves some open questions. In particular, the removal appears to be more effective upon decreasing the plasma excitation energy. This might be due to longer exposure times ( $2 \mathrm{~min}$ instead of $1 \mathrm{~min}$ ) causing sample heating. As the polymer softens with increasing temperature, removal is more
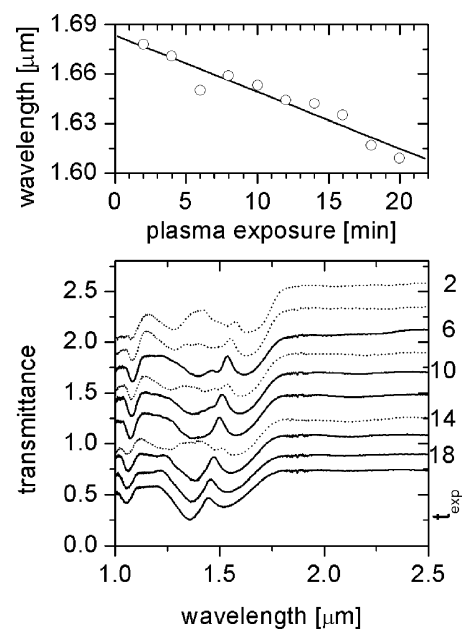

Fig. 6. Top: Position of the reflectance maximum (open circles) and linear fit (solid lines) for exposure to different plasma parameters compared to Fig. 3. Bottom: transmittance spectra for the same sample for increasing plasma exposure time from top to bottom. Two different shapes of spectra are observed (dotted and solid lines), see text for details. Spectra are vertically displaced (exposure time increases from top to bottom) for better clarity. probable. Additionally, it may take some time for the plasma flow to reach its full strength. While missing layers do not influence the tuning, the change in the surface termination will alter the coupling of light to the propagating Bloch-modes inside the photonic crystal [13]. It is interesting to study this effect in detail. The bottom part of Fig. 6 shows the transmittance spectra for increasing plasma exposure (top to bottom), corresponding to the reflectance spectra used to extract the tuning speed. Consider the topmost spectrum (dotted line), which corresponds to 2 min of plasma exposure. The fundamental stop band shows up around $1.64 \mu \mathrm{m}$, directly followed by the side lobes known from the reflectance spectra. In contrast to the reflectance spectra, higher bands can be seen starting below $1.3 \mu \mathrm{m}$. With increasing plasma exposure time, the fundamental stop band as well as the higher bands around $1.1 \mu \mathrm{m}$ shift to the blue. In contrast to this behavior, the side lobes not only shift but also change their strength compared to the fundamental peak periodically. Spectra with pronounced side lobes are plotted in solid lines. As this change in the spectra directly reflects the different coupling to the corresponding modes [13], we conclude, that an intact surface suppresses coupling to the corresponding modes stronger than a corrugated surface. This might be achieved through symmetry breaking on the surface. To further study this behavior, a detailed theoretical analysis is required. To prevent the surface influence on the optical properties, a solid sacrificial layer may be built on top of the photonic crystals.

\section{Conclusions}

In conclusion, we have shown that plasma etching of 3D polymer-based photonic crystals allows for subnanometer tuning of their optical properties. This technique seems to be generally applicable to all kinds of polymer-based photonic crystals and is especially useful for samples prepared with direct laser writing or laser holography, where the smallest feature size (and/ or the filling fraction) is directly limited by the wavelength of light used for exposure. This technique will be very useful to fine tune defect modes with high quality factors to narrow laser lines used to operate functional photonic devices. As small protruding 
features are removed faster, it may also be useful for polishing of wave-guide channels in a $3 \mathrm{D}$ photonic band gap, where surface roughness may cause scattering losses for guided light.

\section{Acknowledgements}

GvF acknowledges support through the Deutsche Forschungsgemeinschaft (DFG) under FR1671/2-1. GAO and SJ are Government of Canada Research Chairs in Materials Chemistry and Theoretical Physics. We are grateful to the Natural Sciences and Engineering Research Council of Canada NSERC for financial support of this work. The group of MW acknowledges support by the DFG through Project A1.4 of the CFN.

\section{References}

[1] E. Yablonovitch, Phys. Rev. Lett. 58 (1987) 2059.

[2] S. John, Phys. Rev. Lett. 58 (1987) 2486.

[3] M. Campbell, D.N. Sharp, M.T. Harrison, R.G. Denning, A.J. Turberfield, Nature 404 (2000) 53.
[4] Y.V. Miklyaev, D.C. Meisel, A. Blanco, G. von Freymann, K. Busch, W. Koch, C. Enkrich, M. Deubel, M. Wegener, Appl. Phys. Lett. 82 (2003) 1284.

[5] S. Kawata, H.B. Sun, T. Tanaka, K. Takada, Nature 412 (2001) 697.

[6] M. Deubel, G. von Freymann, M. Wegener, S. Pereira, K. Busch, C.M. Soukoulis, Nat. Mater. 3 (2004) 444.

[7] S. Wong, V. Kitaev, G.A. Ozin, J. Am. Chem. Soc. 125 (2003) 15589.

[8] M. Deubel, M. Wegener, A. Kaso, S. John, Appl. Phys. Lett. 85 (2004) 1895.

[9] K.M. Ho, C.T. Chan, C.M. Soukoulis, R. Biswas, M. Sigalas, Solid State Commun. 89 (1994) 413.

[10] E. Ozbay, A. Abeyta, G. Tuttle, M. Tringides, R. Biswas, C.T. Chan, C.M. Soukoulis, K.M. Ho, Phys. Rev. B 50 (1994) 1945.

[11] According to Braggs and Snells laws, the position of the maximum in reflectivity $\lambda_{\max }$ is given by $\lambda_{\max }=2 d\left(n_{\text {eff }}^{2}-\right.$ $\left.\sin ^{2} \theta\right)^{1 / 2}$, with $n_{\text {eff }}=f n_{\text {poly }}+(1-f) n_{\text {air }}$, the volume-filling fraction of the polymer $f$, its index of refraction $n_{\text {poly }}$, and the index of refraction $n_{\text {air }}$ of the surrounding medium (air in our case), the interplanar distance $d$, and the angle of incidence $\theta$. A reduction of the volume-filling fraction $f$ therefore directly leads to a blue-shift.

[12] J.F. Galisteo-Lopez, E. Palacios-Lidon, E. Castillo-Martinez, C. Lopez, Phys. Rev. B 68 (2003) 1151091.

[13] G. von Freymann, W. Koch, D.C. Meisel, M. Wegener, M. Diem, A. Garcia-Martin, S. Pereira, K. Busch, J. Schilling, R.B. Wehrspohn, U. Gösele, Appl. Phys. Lett. 83 (2003) 614. 